\title{
Simulating Antigenic Drift and Shift in Influenza A
}

\author{
Nuno Fachada \\ LaSEEB, ISR \\ Instituto Superior Técnico \\ Av. Rovisco Pais \\ 1049-001 Lisboa, Portugal \\ nfachada@laseeb.org
}

\author{
Vitor V. Lopes \\ INETI - Instituto Nacional de \\ Engenharia, Tecnologia e \\ Inovação \\ Estrada do Paço do Lumiar, 22 \\ 1649-038 Lisboa \\ vlopes@laseeb.org
}

\author{
Agostinho Rosa \\ LaSEEB, ISR \\ Instituto Superior Técnico \\ Av. Rovisco Pais \\ 1049-001 Lisboa, Portugal \\ acrosa@laseeb.org
}

\begin{abstract}
Computational models of the immune system and pathogenic agents have several applications, such as theory testing and validation, or as a complement to first stages of drug trials. One possible application is the prediction of the lethality of new Influenza A strains, which are constantly created due to antigenic drift and shift. Here, we present an agent-based model of immune-influenza A dynamics, with focus on low level molecular antigen-antibody interactions, in order to study antigenic drift and shift events, and analyze the virulence of emergent strains. At this stage of the investigation, results are presented and discussed from a qualitative point of view against recent and generally recognized immunology and influenza literature.
\end{abstract}

\section{Categories and Subject Descriptors}

\section{I.6.3 [Simulation and Modeling]: Applications}

\section{Keywords}

Immune System, Influenza A, Simulation, Agent-based Modeling

\section{INTRODUCTION}

There are several approaches to immune system (IS) and pathogen modeling [23], among which models based on differential equations are probably the most common [16]. This methodology is mostly used for modeling particular aspects of the IS and pathogens, among which is its use on the study of influenza dynamics [2, 8, 20] and treatment [5].

Agent-based modeling (ABM) techniques are well suited for describing the diversity of IS and pathogen dynamics, taking into account behavior distribution [22], simplified insertion of new entities or substances and natural consideration of non-linear interactions between agents, capturing emergent phenomena [9]. Most agent-based approaches model a generic IS [7, 15, 21, 24, 29], and use such model

Permission to make digital or hard copies of all or part of this work for personal or classroom use is granted without fee provided that copies are not made or distributed for profit or commercial advantage and that copies bear this notice and the full citation on the first page. To copy otherwise, to republish, to post on servers or to redistribute to lists, requires prior specific permission and/or a fee.

SAC'09 March 8-12, 2009, Honolulu, Hawaii, U.S.A.

Copyright 2009 ACM 978-1-60558-166-8/09/03 ...\$5.00. to simulate particular situations (e.g., HIV infection [10, 17, 18], pulmonary tuberculosis [31]) or test specific theories (e.g. immunological memory [26]). Some models specifically target particular contexts; such is the case of the models developed by C. Beauchemin, used for the study of influenza A infections. In [6], the influence of the disease's spatial distribution is investigated using a simple model capable of reproducing the virus basic dynamical features. The initial distribution of infected cells, regeneration rate of dead cells and proliferation rate of immune cells are further investigated in [3]. The same model was also used to reproduce the dynamics of in vitro infection with influenza A [4], effectively combining in vitro and in silico tools.

In this paper we describe an immune-influenza A agentbased model, with emphasis on low level molecular antigenantibody interactions. The model was developed using the LAIS simulator [13], and can be divided in three parts: a) epithelium, a tissue composed of epithelial cells, subject to infection by influenza A; b) IS cells, which work together in order to remove infections; and c) influenza A virions. Agents are modeled at the cellular (epithelial and IS cells) or virion (influenza A) level. The IS submodel has been successfully used in the simulation of bacterial attack and vaccine administration, demonstrating memory, specificity and specialization features [14]. In the present case, the aim is to study antigenic drift and shift events in influenza A, and analyze the virulence of emergent strains. The main results of this work serve to clarify: a) the fine balance of influenza antigens in a successful infection; b) the role of specific IS properties in dealing with influenza infection; c) the difficulties antigenic drift can pose on the IS response; and d) the potential dangers of antigenic shift. Hopefully, this type of models could serve a useful purpose in predicting the virulence of annual influenza epidemics, and more importantly, the uprising of a lethal influenza pandemic due to antigenic shift. In section 2 the influenza virus is presented from human and physiopathological points of view. The LAIS simulator and the immune-influenza A model are described in sections 3 and 4, respectively. In section 5 simulations and results are presented and compared with immunology and influenza theory from literature. Finally, in section 6 we conclude the presented work, underlining the model's potential, envisaging possible forms of solidifying the produced results.

\section{THE INFLUENZA VIRUS}

Influenza is caused by a virus that attacks mainly the upper respiratory tract, leading to symptoms such as high 
fever, myalgia and sore throat, among others. Most people recover without requiring any medical treatment; however, in high-risk groups (e.g. the elderly) the infection may lead to severe complications of underlying diseases, pneumonia and death. The disease spreads quickly at a global level in seasonal epidemics, with significant economic impact. Annual epidemics are estimated to result in three to five million cases of severe illness and between 250,000 and 500,000 deaths worldwide [32].

Among the several types of influenza virus which attack humans, type $\mathrm{A}$ has the highest mutation rate and causes the most aggressive symptoms, being responsible for all known influenza pandemics [19, 28, 32].

\subsection{Physiopathology}

Influenza, as most viruses, takes over the replicative machinery of host cells in order to grow and reproduce, with cunning strategies to evade immune defenses. More specifically, influenza virus infects epithelial cells of the respiratory tract.

Cytotoxic T cells (Tc) are crucial in the elimination of viral infection, killing infected cells via recognition of a complex formed by viral antigenic peptide and MHC class I self molecules [1, 27]. The release of $\mathrm{IFN}-\gamma$ by Helper $\mathrm{T}$ cells (Th) is another important aspect of the immune response, promoting resistance to viral subversion. Usually, in a primary viral encounter, the defensive role of antibody is diminished, as viral particles take shelter in the intracellular habitat, mostly infecting adjacent cells, thus limiting their extracellular exposure. When an antibody response is initiated, the infection is either widespread or controlled by cell-mediated mechanisms. However, antibody plays an important part in preventing reinfection: in secondary challenges specific memory B cells exist and are ready to produce high affinity antibodies which will bind and neutralize free virions before they gain entrance into host cells.

One of the strategies the influenza virus uses to escape detection is changing antigens by drift or shift. In the first case, drift occurs through point mutations in the viral genome during replication, which can lead to a loss of immunity from previously existing antibodies. Major antigen changes take place during antigenic shift, which can occur when two viruses simultaneously infect a host cell; the infected cell acts as a "mixing vessel", producing a new virus composed of genome segments belonging to the original viruses [33]. Influenza A displays both these strategies; antigenic drift causes seasonal influenza epidemics, while antigen shift, although rarer, may cause serious influenza pandemics [19]. Influenza A carries two surface antigens, hemagglutinin (HA) and neuraminidase (NA), both of which recognize the same host receptor, sialic acid [30]. HA and NA define the virus subtype accordingly to their reactivity [33]. Some well know subtypes, due to their lethality, are H1N1 (Spanish flu, 1918), H2N2 (Asian flu, 1957), H3N2 (Hong Kong Flu, 1968) and H5N1 (avian flu, current pandemic threat) [19, 28]. HA binds sialic acid, a necessary first step for gaining entry into the host cell; NA on the other hand cleaves sialic acid to facilitate the release of progeny virus and promote the spread of infection. A successful infection depends, among other factors, on the balance of these two antigens [30].

\section{LAIS SIMULATOR}

The LAIS simulator is a multithreaded agent-based sim- ulation platform, offering a set of tools for the simulation of biological systems. The platform is implemented in Java and makes use of following open source libraries: a) the Repast Agent Simulation Toolkit [25] classes that provide or simplify spatial organization and visualization, event scheduling and simulation output (e.g., charts, CSV files, movies); and b) the Simple XML serialization library ${ }^{1}$ that provides simple class development and instantiation using XML.

The two main actors in the LAIS framework are substances and agents. The simulator is organized in two layers: a) a specialized cellular automaton (CA) responsible for substance diffusion, reaction and degradation; and, b) the agent layer where the agents move and act. The communication between these layers occurs when agents produce or consume substances, or when an agent action depends on the underlying substances. Current implementation restricts the simulation to discrete time and two-dimensional space. The multithreading capabilities of the simulator work by equally distributing the CA cells through a number of threads dependent on the number of available processor cores; this is possible because during a simulation step, each CA cell becomes an independent processing unit [13].

\subsection{Substances}

Substances are uniquely identified by a 64 bit string, allowing a repertoire of $2^{64} \approx 10^{19}$ different substances. In the model specification it is possible to attribute specific biological functions to different bit substrings. The biological affinity between substances primarily depends on the existence of complementary zones, i.e., regions where the biological substances can "fit" with each other. To mimic the IS, the bit string of substances that model IS antibodies are composed by: a) a constant region responsible for secondary functions such as macrophage binding or complement fixation, and b) a variable region which is used to determine the binding affinity with antigen. The biological affinity is implemented by the Hamming distance between two substance bitstrings [11].

LAIS represents substances as real valued concentrations, allowing to: a) model diffusion and reaction phenomena in the CA layer; and b) simulate the substances present on the agent surface, in the agent layer. Antigens are modeled as substances and thus differentiated from pathogenic agents themselves.

New substances can be dynamically created during simulation as the result of: a) different substances produced by mutation of cloning agents; and, b) substance merging. Merging can be either affinity dependent, such as in the antigen-antibody complex formation or independent, such as in the case of the complex formed by MHC and the antigen peptide.

The simulator offers the possibility to group the substances into families in order to: a) simplify the process of tracking substances with similar functions, e.g. in B cell response, where a multitude of different antibodies are temporarily produced; and, b) allow the definition of substance merging rules affecting specific families. In the latter case, model specification is considerably facilitated and the substance merging simulation becomes computationally feasible.

\subsection{Agents}

$\overline{{ }^{1} \text { http://simple.sourceforge.net/ }}$ 


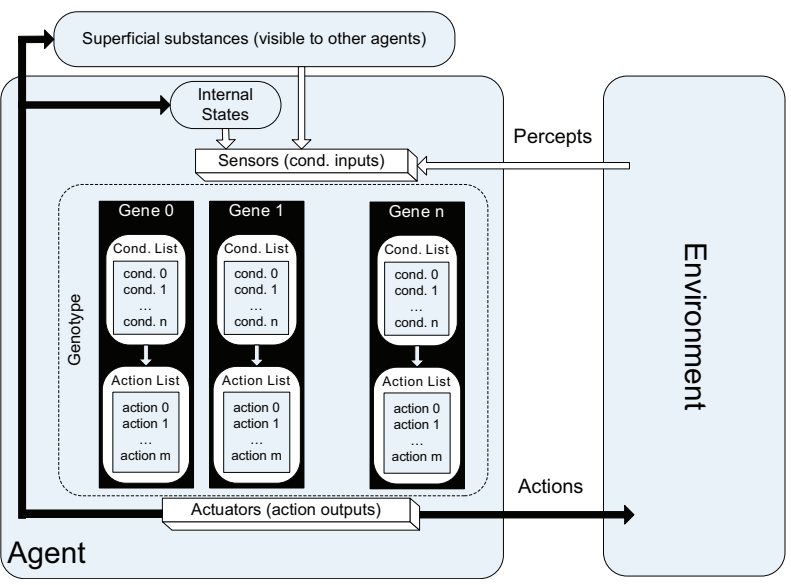

Figure 1: The LAIS agent model.

Agents have a set of conditions which evaluate state, superficial substance concentration and the local CA cell, analyzing local substance concentration, as well as substances displayed by other agents. These conditions are grouped in lists of conditions; each list of conditions is associated to a list of actions. A "condition list - action list" mapping is called a "gene". In order to perform the actions in a list, all the conditions in the associated condition list must yield true. Fig. 1 shows the schematics of a LAIS agent. Conditions and actions are hard-coded Java classes, but accept instantiation parameters, making them flexible. The grouping of conditions and actions with different instantiation parameters permits a vast range of behaviors. If a particular behavior cannot be achieved using available conditions and actions, it is relatively simple to code additional ones, following specific interfaces. The agent set of genes can be referred as the agent's genotype. Agent movement is controlled by these conditions; movement can be random, inertial (higher probability of moving forward) or substance dependent (simulating chemotaxis, cell movement directed by a chemical concentration gradient [1]).

Evolution takes place when an agent creates another agent, either by a cloning process (e.g., cellular division) or by producing a different type of agent (e.g., an infected immune cell producing viruses); these processes are carried away by actions defined in the agent's genetic code. In such cases, conditions and actions are also cloned. These have a mutation parameter which can modify referenced substances; because the IS and pathogens interact via superficial substances, this mechanism forms the basis for IS-pathogen coevolution, namely IS recognition of previously unknown pathogens and pathogen evasion from IS detection. LAIS also supports the exchange of genetic code (at gene level) [17], adding another level of complexity for modeling realistic evolving systems.

\section{MODEL DESCRIPTION}

One of the most complex challenges when developing a IS model is to find a balance between scale, granularity and computational feasibility. Features that are included in the model should not only be theoretically and experimentally sound, but also relevant in the context of the simulations to perform. Knowledge gaps, incomplete data and excluded system features imply that models are incomplete, always abstract to some point. However, an incomplete model can still do a good job of simulating reality. Biological systems can also work without various parts; they are robust, having redundant features and components with overlapping functions. As such, if a model captures the principal components of a biological system, there is no reason why it cannot yield realistic simulations [12].

The model here presented includes several distinct entities and substances which approximately reflect aspects we consider the most relevant of immune-influenza A dynamics. Tables 1 and 2 respectively summarize characteristics of agents and substances included in the model. A complete model description, which includes the genetic code of the agents, is available in [13].

\section{SIMULATIONS AND RESULTS}

The simulations presented in this section were performed using a 14x14 toroidal hexagonal grid. During a steady-state phase, were no infection or IS response is taking place, the number of distinct agents varies between 400 and 500. At peak immune responses, the number of distinct agents can reach $2 \times 10^{5}$.

These simulations yielded a considerable quantity of data, of which we present and interpret the most relevant, either in the form of graphs or textual descriptions during analysis and discussion of simulations.

\subsection{Influenza subtypes and antigenic drift}

The balance of sialic acid affinity between HA and NA antigens is of major importance for efficient virus replication [30]. In this experiment, four simulations are performed, each with the deployment of one influenza subtype at ticks 50 and 500. Each subtype has a different set of superficial antigens, with specific affinities for sialic acid (the superficial receptor of epithelial cells). Table 3 shows the different antigens bit string and affinity with sialic acid (whose bit string is 04D2h), prior to mutation; only the 16 less significant bits are relevant for this affinity; bits 16 to 31 (in italic) are used to determine affinity with TCR when antigen is complexed with MHC. These affinity values are merely demonstrative, and do not correlate with sialic acid affinity of "real" HA and NA types.

The four antigens presented in table 3 allow to experiment with four influenza subtypes: H1N1, H1N2, H2N1 and H2N2. Again, these do not correlate with the real subtypes.

\subsubsection{Analysis of results}

The H1N1 subtype has high affinity HA and NA, meaning it can rapidly infect cells (due to HA), and proliferate in larger quantities (due to NA). In principle, this should be the most lethal strain. However, that is not the case. Its rapid proliferation, killing and infecting epithelial cells too fast (fig. 2), limits its own expansion; the sudden high number of extracellular virions draws the attention of humoral mechanisms; the presence of a large number of locally infected cells provokes a strong Th cell reaction, due to B cell and APC antigen presentation; this leads to the production of IFN- $\gamma$ (limiting the spread of the infection) and the activation of Tc cells; these destroy locally infected epithelial cells, putting a definitive stop to the viral infection. In its first strike, the H1N1 strain lasted approximately 215 ticks, with a maximum of 80 simultaneous epithelial infections (fig. 
Table 1: Agents included in immune-influenza A model.

\begin{tabular}{lll}
\hline Agent & Type & Behavior \\
\hline Influenza A & Pathogen (virus) & $\begin{array}{l}\text { Infect and insert genetic code in epithelial cells, forcing them to produce in- } \\
\text { fluenza A virions; infection is dependent on HA antigen-sialic acid affinity, while } \\
\text { virion release is dependent on NA antigen-sialic acid affinity. }\end{array}$ \\
$\begin{array}{lll}\text { Epithelial cell } \\
\text { Tc cell }\end{array}$ & $\begin{array}{l}\text { Epithelium } \\
\text { Immune System }\end{array}$ & $\begin{array}{l}\text { Kill infected cells (i.e., cells displaying MHC Class I + antigenic peptide com- } \\
\text { plex). }\end{array}$ \\
Th cell & Immune System & $\begin{array}{l}\text { Produce substances which assist other immune cells. } \\
\text { B cell }\end{array}$ \\
APC cell & Immune System & $\begin{array}{l}\text { Produce antibody; present antigen to Th cells via MHC Class II. } \\
\text { Immune System } \\
\text { Phagocyte (ingest) pathogenic agents and antigens, with greater probability if } \\
\text { pathogen is covered with antibody (opsonized); present antigen to Th cells via } \\
\text { MHC Class II. }\end{array}$ \\
\hline
\end{tabular}

Table 2: Substances included in immune-influenza A model.

\begin{tabular}{|c|c|c|c|}
\hline Substance & Source & Location & Effector function \\
\hline Interferon- $\gamma$, IFN- $\gamma$ & Th, Tc & Local & $\begin{array}{l}\text { Inhibits sialic acid production in epithelial cells, pro- } \\
\text { tecting them from infection; increases probability of } \\
\text { epithelial cell apoptosis. }\end{array}$ \\
\hline Interleukin-2, IL-2 & Th, Tc & Local & Induces proliferation of activated Th, Tc and B cells. \\
\hline Interleukin-12, IL-12 & $\mathrm{APC}$ & Local & Induces Th proliferation and IFN- $\gamma$ production. \\
\hline Antibodies (family) & $\mathrm{B}$ & Local, Superficial & $\begin{array}{l}\text { Variable region binds antigen depending on affinity; } \\
\text { constant region }(\mathrm{Fc}) \text { binds } \mathrm{Fc} \text { receptors on APC cells. }\end{array}$ \\
\hline Antigens (family) & Influenza A & Local, Superficial & $\begin{array}{l}\text { HA antigens bind sialic acid promoting infection, NA } \\
\text { antigens cleave sialic acid promoting virion release. }\end{array}$ \\
\hline MHC Class I & Epithetial cells & Superficial & $\begin{array}{l}\text { When complexed with antigenic peptide, can bind } \\
\text { TCR CD } 8 \text { in Tc cells, depending on affinity. }\end{array}$ \\
\hline MHC Class II & $\mathrm{APC}, \mathrm{B}$ & Superficial & $\begin{array}{l}\text { When complexed with antigenic peptide, can bind } \\
\text { TCR CD } 4 \text { in Th cells, depending on affinity. }\end{array}$ \\
\hline TCR CD8 & $\mathrm{Tc}$ & Superficial & $\begin{array}{l}\text { Binds MHC Class I }+ \text { antigenic peptide complex } \\
\text { present on the surface of infected epithelial cells, pro- } \\
\text { moting the destruction of such cells. }\end{array}$ \\
\hline TCR CD4 & Th & Superficial & $\begin{array}{l}\text { Binds MHC Class II }+ \text { antigenic peptide complex } \\
\text { present on the surface of APCs and B cells, promoting } \\
\text { assistance to such cells. }\end{array}$ \\
\hline Fc receptor & $\mathrm{APC}$ & Superficial & $\begin{array}{l}\text { Binds antibody constant region, promoting more effi- } \\
\text { cient phagocytosis. }\end{array}$ \\
\hline Sialic acid & Epithetial cells & Superficial & Recognized by HA and NA influenza antigens. \\
\hline
\end{tabular}

Table 3: Influenza antigens bit string and affinity with sialic acid.

\begin{tabular}{cccc}
\hline Antigen & \multicolumn{2}{c}{ Bit string } & Affinity \\
\hline HA1 & 0707 & 7B21h & 0.8125 \\
HA2 & 1616 & 0622h & 0.3125 \\
NA1 & 2D2D & 1B2Dh & 0.8125 \\
NA2 & 3C3C & 0BDDh & 0.5000 \\
\hline
\end{tabular}

2).

The H1N2 and H2N1 strains proved to be more infectious, lasting about 275 ticks in their first strike (figs. 3 and 4, respectively). H1N2 can infect cells quickly (high affinity HA), but the number of released virions is low (low affinity NA), resulting in a maximum of less than 20 virions simultaneously present in the extracellular milieu; however, a maximum of 80 simultaneous epithelial infections indicates this is not an handicap, on the contrary, the low virus titer allows it to remain concealed from humoral mechanisms, with barely detectable Th and B cell responses. As a consequence, little humoral memory was created. In the second strike, the strain provokes a state of chronic illness lasting a record value of 390 ticks, the highest value of all eight infections (four primary, four secondary), with virus titer never reaching the 30 unit mark. On the other hand, the maximum viral titer in the primary $\mathrm{H} 2 \mathrm{~N} 1$ infection is of 46 units (the highest of all eight infections), but correspondently provokes the most powerful humoral response (figs. 8); the immune memory created quickly eliminated the strain during the second strike, lasting no more than 130 ticks.

The H2N2 strain, with low affinity HA and NA, proved to be the least resistant and the least infectious (fig. 5).

\subsubsection{Balance between HA and NA antigens}

This experiment demonstrated the fine balance between HA and NA antigens and their importance in a successful infection, as discussed in [30]. A high affinity HA antigen seems to be more important for kick starting the infection, while NA functions like a throttle, determining the number 


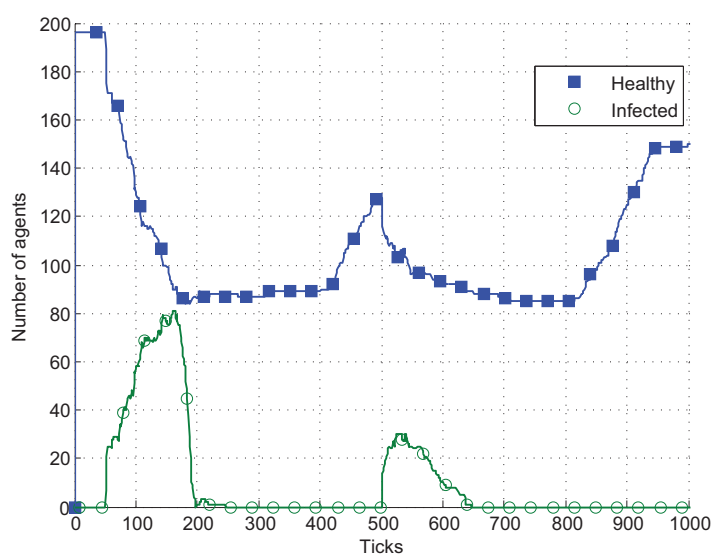

Figure 2: Healthy and infected epithelial cells during challenge from $\mathrm{H} 1 \mathrm{~N} 1$ subtype.

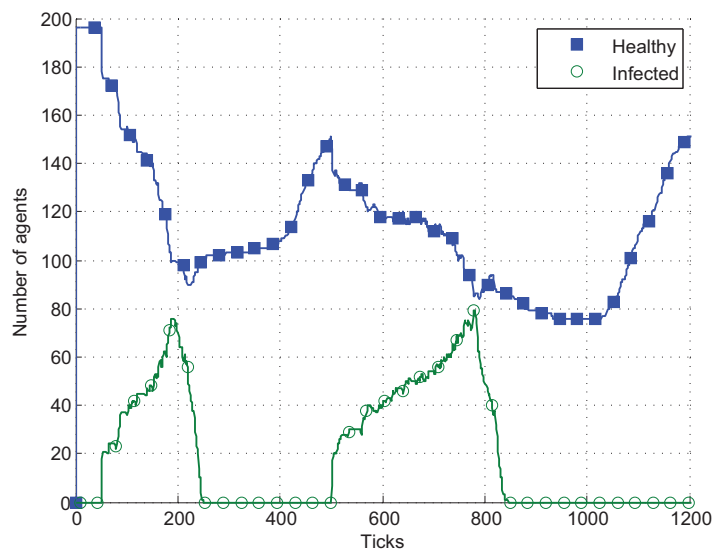

Figure 3: Healthy and infected epithelial cells during challenge from H1N2 subtype.

of released virions. As observed in the simulations, high affinity NA is probably not an optimum companion for high affinity HA. More testing is required in order to better explore HA-NA balance, and its exact effect on the success of an infection. Such a study must take into account that influenza strains may have other infectivity parameters, and that antigenic drift causes noise which can difficult the analysis of results. In this last case, the solution is to perform a greater number of tests for the same parameters, in order to obtain more statistically significant results.

\subsubsection{Effects of antigenic drift in immune response, immune memory and antibody effectiveness}

Antigenic drift can be asserted indirectly by considering the number of different antigens present in the simulation environment. The IS tries to respond with the production of a larger diversity of antibody, in a reaction to the stimulus from the many different antigens.

In all cases, except for H1N2, immune memory was acquired against the invading strain. However, the effectiveness of memory is reduced when compared to a simulation of non-mutating bacteria using the same simulation platform and basic IS model [14]; in this case, it was possible to ob-

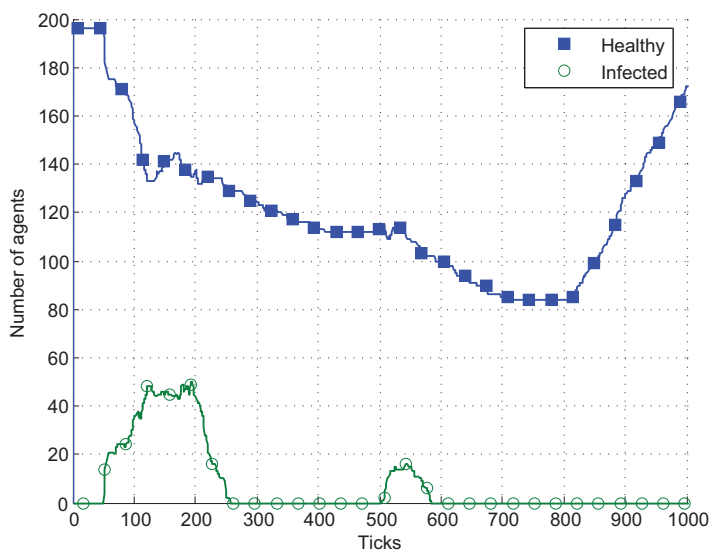

Figure 4: Healthy and infected epithelial cells during challenge from H2N1 subtype.

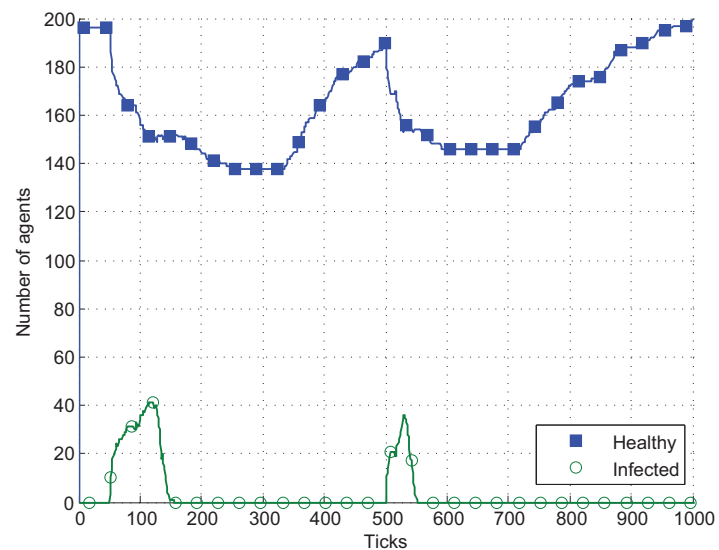

Figure 5: Healthy and infected epithelial cells during challenge from H2N2 subtype.

serve a relatively high degree of antigen-antibody complex; this occurs because B cells are stimulated by a single antigen, resulting in plasma cells which produce high affinity antibody; this antibody binds large quantities of antigen, resulting in also large quantities of antigen-antibody complex. In the case presented here, figs. 6, 7, 8 and 9 show that very small quantities of antigen-antibody complex are produced. This happens due to antigenic drift; each newly created influenza virion can be different from its precursor, deploying different types of antigen, not yet known to the latest versions of antibody [27].

\subsubsection{Role of Tc cells}

As described in literature, the average number of Tc cells during an immune response is one order of magnitude above the number of Th cells [1], which was confirmed by the obtained results. Sudden drops in infected epithelial numbers, visible in figs. $2,3,4$ and 5 , occur practically at the same time when effector Tc cells begin to be massively produced, thus demonstrating their cytotoxic function.

\subsubsection{Estimate of temporal resolution}

With the data gathered so far, it is possible to perform an 


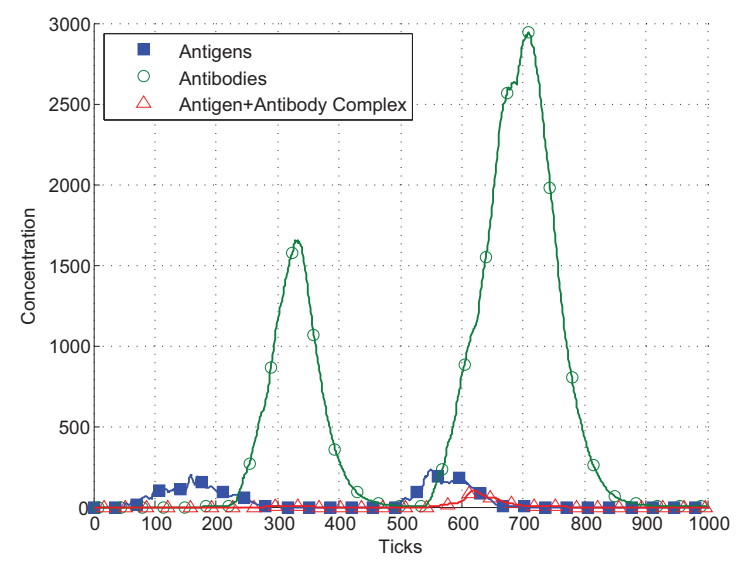

Figure 6: Substance family concentration during challenge from H1N1 subtype.

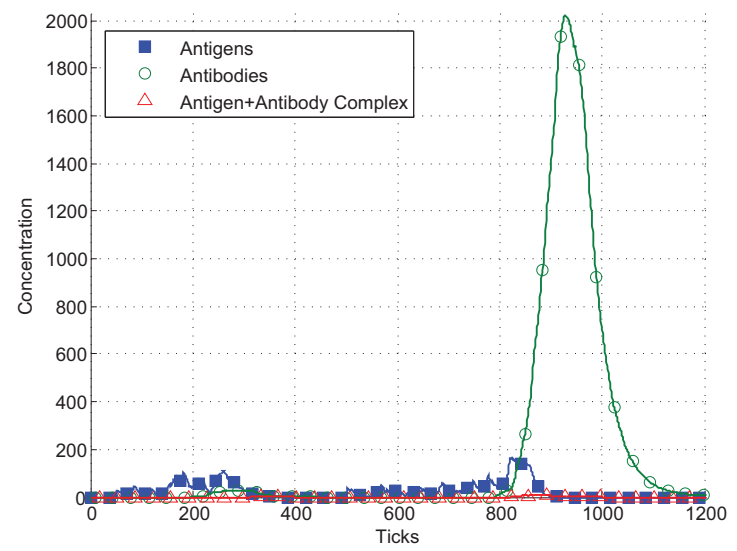

Figure 7: Substance family concentration during challenge from H1N2 subtype.

estimate of the temporal resolution of the simulations. In [27], it is suggested that the appearance of specific antibody occurs about 6 days after initial influenza infection. In our simulations, specific antibody appears in average 150 ticks after initial infection (figs. 6, 7, 8 and 9), indicating about 25 ticks per day temporal resolution. A similar result can be obtained by another path: a typical influenza infection usually lasts for about a week, with most people recovering within one to two weeks [32], so let's consider an eight day infection period. The four primary infections have an average duration of 220 ticks (not shown), producing a similar temporal resolution estimate of 27.5 ticks per day.

\subsection{Antigenic shift}

Antigenic shift is a major antigenic change which occurs at irregular intervals, being the main responsible for influenza pandemics. In the influenza virus family, only influenza A is susceptible to this event. Antigenic shift can lead to the evolution of new human influenza A virus through the acquisition of a new HA gene encoding a different subtype from an avian influenza, or by the adaptation of an avian virus, causing it to become transmissible between humans [19].

In this experiment we infect the host with two different

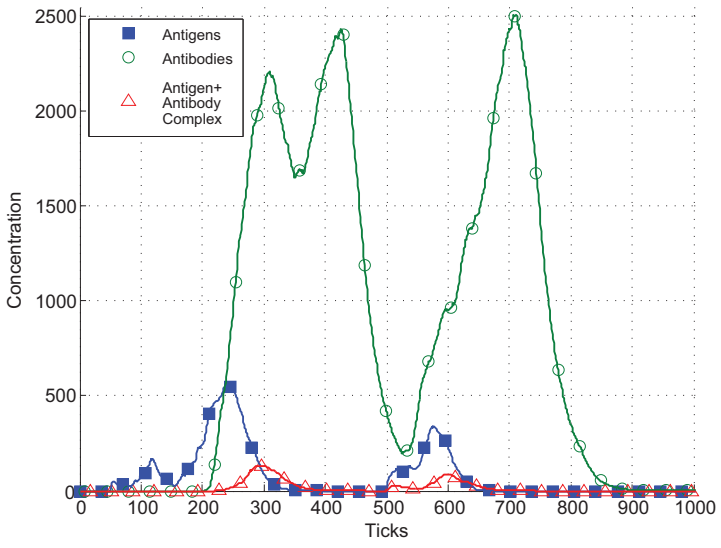

Figure 8: Substance family concentration during challenge from $\mathrm{H} 2 \mathrm{~N} 1$ subtype.

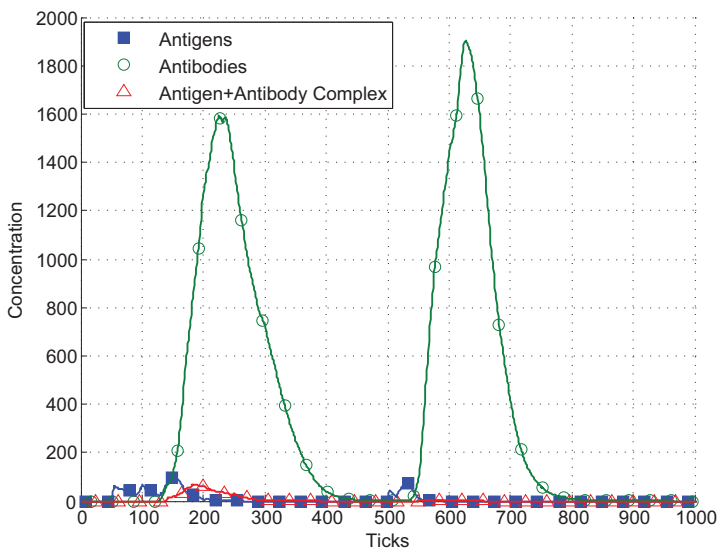

Figure 9: Substance family concentration during challenge from H2N2 subtype.

influenza strains, H1N2 and H2N1, which were studied separately in the previous subsection. The goal is to verify the occurrence of antigenic shift, and if it leads to the creation of a more infectious subtype.

At tick 50, 25 units of $\mathrm{H} 1 \mathrm{~N} 2$ and 25 units of $\mathrm{H} 2 \mathrm{~N} 1$ are deployed in the simulation environment. Contrary to what was verified in the previous subsection, H2N1 seems to adapt better in a competitive situation; as can be verified in fig. $10, \mathrm{H} 2 \mathrm{~N} 1$ virion titer is constantly superior to that of $\mathrm{H} 1 \mathrm{~N} 2$.

The appearance of H1N1 subtype confirms the existence of antigenic shift; at tick 175, the emergent H1N1 subtype experiences a fast rise, surpassing the impact of the H1N2 subtype, which never really takes off, as opposed to what was observed in the previous experience. However, the emergent H1N1 subtype does not become more infectious than the original H1N2, which becomes the IS main adversary. The overall infection was eliminated in 250 ticks, slightly faster than what was verified in some of the previous cases. The higher number of initially inserted virions would suggest that this combination is "weaker" than the insertion of isolated strains; however, the elevated number of viral titter may also have conducted to a faster humoral response, ac- 


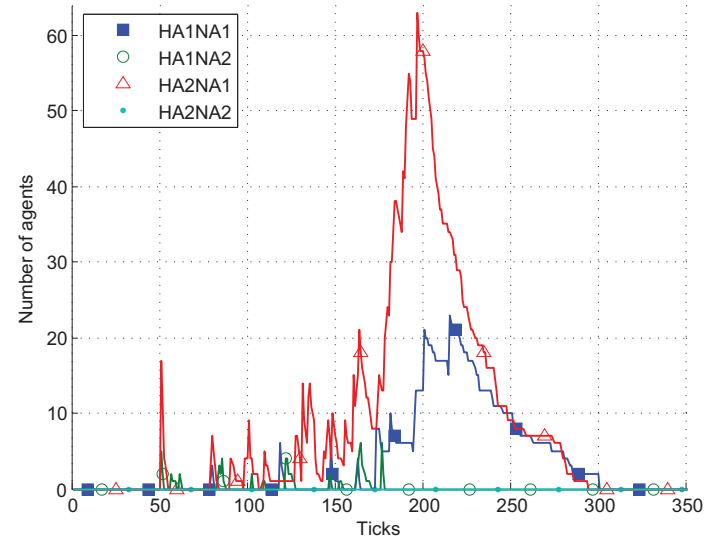

Figure 10: Evolution of Influenza A subtypes during antigenic shift simulation.

celerating the removal of infection; this is corroborated by fig. 12, which indicates a 100 tick interval between virion insertion and beginning of humoral response; in the previous cases this value was closer to 150 ticks (figs. 6 and 8 ). The $\mathrm{H} 2 \mathrm{~N} 1$ original strain reached a maximum value of 65 units of circulating virion, fig. 10, higher than any of the previous cases. From ticks 150 to 190, there were more infected epithelial cells than healthy ones (fig. 11), a situation which never occurred while the subtypes were studied in isolation.

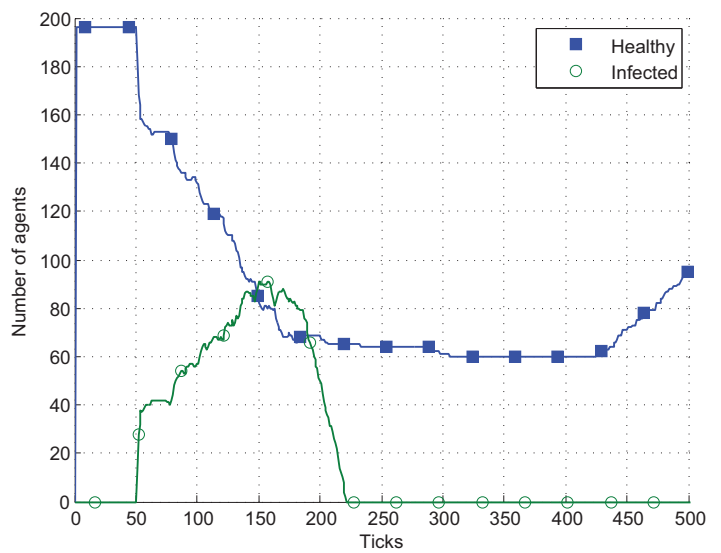

Figure 11: Evolution of epithelial cells during antigenic shift simulation.

When the B cell response takes place, cells undergoing somatic hypermutation do not often become plasma cells; in fact, plasma cell number barely surpasses half the number of cells in somatic hypermutation, confirming the difficulty in keeping pace with continuously mutating and recombining antigens. This is further verified in fig. 12, showing elevated antigen diversity (the highest in all influenza simulations). Production of antigen + antibody complex was very low, in spite of the variety of produced antibodies.

Several Tc cells become activated at tick 75 due to recognition of MHC Class I + antigen complex presented by epithelial cells; however, they lack the ability of becoming effector without the help of Th cells, which only occurs from tick 150 onward, when the humoral response takes place. At this stage, IFN- $\gamma$ kicks in, slowing the spread of infection; a few effector Tc cells also begin to eliminate infected epithelials. At tick 200, a spike in Tc clonal expansion and effector production results in the rapid extermination of infected cells (fig. 11); when the remaining virion infect healthy cells, the massive quantity of Tc effector clones immediately eliminates such cells, severely limiting the replication capabilities of the virus. After the infection is fully removed and IFN- $\gamma$ dissipated, epithelial cells begin to populate epithelial free spaces.

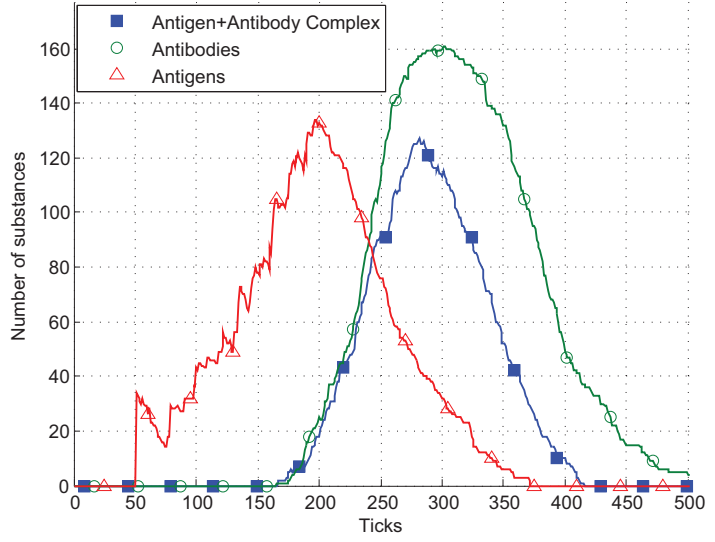

Figure 12: Substance family diversity during antigenic shift simulation.

\section{CONCLUSIONS}

Simulation results were within our initial expectations. A novel simulation of immune-influenza A dynamics was developed and successfully tested against theoretical data from literature - unfortunately, this area of investigation lacks comprehensive experimental data. The antigenic drift simulations demonstrated the importance of HA and NA balance in a successful infection, while clarifying the role of specific IS cells in the immune response; the difficulties antigenic drift can pose on the IS response were also asserted. In the antigenic shift experiment, though the event was observed, the appearance of a lethal strain was not verified. To further study this event, more subtypes should be taken into account and other infectivity parameters, such as virion extracellular survival time, should be considered.

Given the stochastic nature of these simulations and considering the complexity of simultaneous antigenic drift and shift, each set of parameters should be tested a sufficient number of times in order to be adequately explored from a statistical point of view. Nonetheless, the achieved results demonstrate that this model could be a starting point for predicting the impact of influenza epidemics and the probability of pandemic outbreak.

\section{ACKNOWLEDGMENTS}

This work was partially supported by Fundação para a Ciência e a Tecnologia (ISR/IST plurianual funding) through the POS_Conhecimento Program that includes FEDER funds. 


\section{REFERENCES}

[1] A. Abbas and A. Lichtman. Basic immunology: functions and disorders of the immune system. Saunders Elsevier, 2nd edition, updated edition 2006-2007 edition, 2006.

[2] P. Baccam, C. Beauchemin, C. Macken, F. Hayden, and A. Perelson. Kinetics of Influenza A Virus Infection in Humans. Journal of Virology, 80(15):7590-7599, 2006.

[3] C. Beauchemin. Probing the effects of the well-mixed assumption on viral infection dynamics. Journal of Theoretical Biology, 242(2):464-477, 2006.

[4] C. Beauchemin, S. Forrest, and F. Koster. Modeling Influenza Viral Dynamics in Tissue. Lecture Notes in Computer Science, 4163:23-36, 2006.

[5] C. Beauchemin, J. McSharry, G. Drusano, J. Nguyen, G. Went, R. Ribeiro, and A. Perelson. Modeling amantadine treatment of influenza $\mathrm{A}$ virus in vitro. Journal of Theoretical Biology, 254(2):439-451, 2008.

[6] C. Beauchemin, J. Samuel, and J. Tuszynski. A Simple Cellular Automaton Model for Influenza A Viral Infections. Journal of Theoretical Biology, 232(232):223-234, 2005.

[7] M. Bernaschi and F. Castiglione. Design and implementation of an immune system simulator. Computers in Biology and Medicine, 31(5):303-331, 2001.

[8] G. Bocharov and A. Romanyukha. Mathematical model of antiviral immune response. III. Influenza A virus infection. J Theor Biol, 167(4):323-360, 1994.

[9] E. Bonabeau. Agent-based modeling: Methods and techniques for simulating human systems. Proceedings of the National Academy of Sciences, 99(3):7280-7287, May 2002.

[10] F. Castiglione and M. Bernaschi. HIV-1 Strategies of Immune Evasion. International Journal of Modern Physics C, 16(12):1869-1878, 2005.

[11] F. Celada and P. Seiden. A Computer Model of Cellular Interactions in the Immune System. Immunology Today, 13(2):56-62, 1992.

[12] I. Cohen. Modeling Immune Behavior for Experimentalists. Immunological Reviews, 216(1):232-236, 2007.

[13] N. Fachada. Agent-based Simulation of the Immune System. Master's thesis, Instituto Superior Técnico, Lisboa, July 2008.

[14] N. Fachada, V. V. Lopes, and A. Rosa. Simulation of Immune Response to Bacterial Challenge. In Proceedings of the 22nd annual European Simulation and Modelling Conference, pages 252-257. Eurosis, October 2008.

[15] V. Folcik, G. An, and C. Orosz. The Basic Immune Simulator: An agent-based model to study the interactions between innate and adaptive immunity. Theoretical Biology and Medical Modelling, 4(39), September 2007.

[16] S. Forrest and C. Beauchemin. Computer Immunology. Immunological Reviews, 216(1):176-197, 2007.

[17] A. Grilo, A. Caetano, and A. Rosa. Agent based Artificial Immune System. In Proc. GECCO-01, Vol. LBP, pages 145-151, 2001.
[18] Z. Guo, H. K. Han, and J. C. Tay. Sufficiency Verification of HIV-1 Pathogenesis based on Multi-Agent Simulation. In Proceedings of the 2005 conference on Genetic and Evolutionary Computation, pages 305-312. ACM Press New York, NY, USA, 2005.

[19] A. Hampson and J. Mackenzie. The influenza viruses. Medical Journal Australia, 185(10 Suppl):S39-S43, 2006.

[20] B. Hancioglu, D. Swigon, and G. Clermont. A dynamical model of human immune response to influenza A virus infection. Journal of Theoretical Biology, 246(1):70-86, 2007.

[21] S. Kleinstein and P. Seiden. Simulating the Immune System. Computing in Science and Engineering, 2(4):69-77, 2000.

[22] B. Kohler, R. Puzone, P. Seiden, and F. Celada. A systematic approach to vaccine complexity using an automaton model of the cellular and humoral immune system. i. viral characteristics and polarized responses. Vaccine, 19(7-8):862-76, 2000.

[23] Y. Louzoun. The Evolution of Mathematical Immunology. Immunological Reviews, 216(1):9-20, 2007.

[24] M. Meier-Schellersheim and G. Mack. SIMMUNE, a tool for simulating and analyzing immune system behavior. Technical report, Institut fr Theoretische Physik, Universitt Hamburg, 1999.

[25] M. North, N. Collier, and J. Vos. Experiences Creating Three Implementations of the Repast Agent Modeling Toolkit. ACM Transactions on Modeling and Computer Simulation, 16(1):1-25, 2006.

[26] M. Robbins and S. Garrett. Evaluating Theories of Immunological Memory Using Large-Scale Simulations. In C. Jacob, M. L. Pilat, P. J. Bentley, and J. Timmis, editors, Artificial Immune Systems, volume 3627 of Lecture Notes in Computer Science, chapter 16, pages 193-206. Springer Berlin / Heidelberg, 2005.

[27] I. M. Roitt and P. J. Delves. Essential Immunology. Blackwell Publishing, 10th edition, 2001.

[28] K. Ryan and C. Ray. Sherris Medical Microbiology: An Introduction to Infectious Diseases. McGraw-Hill Medical, 2004.

[29] J. Tay and A. Jhavar. CAFISS: a Complex Adaptive Framework for Immune System Simulation. In Proceedings of the 2005 ACM symposium on Applied Computing, pages 158-164. ACM Press New York, NY, USA, 2005.

[30] R. Wagner, M. Matrosovich, and H. Klenk. Functional balance between haemagglutinin and neuraminidase in influenza virus infections. Rev. Med. Virol., 12:159-166, 2002.

[31] C. Warrender. Modeling intercellular interactions in the peripheral immune system. $\mathrm{PhD}$ thesis, The University of New Mexico, 2004.

[32] World Health Organization. http://www.who.int/.

[33] M. Zambon. Epidemiology and pathogenesis of influenza. Journal of Antimicrobial Chemotherapy, 44:3-9, 1999. 